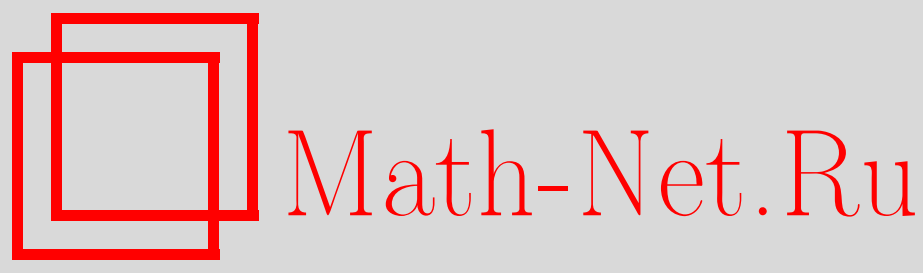

Ю. Хонконен, Контурно упорядоченные функции Грина в стохастической теории поля, ТМ $\Phi, 2013$, том 175 , номер 3, 455-464

DOI: https://doi.org/10.4213/tmf8483

Использование Общероссийского математического портала Math-Net.Ru подразумевает, что вы прочитали и согласны с пользовательским соглашением http://www.mathnet.ru/rus/agreement

Параметры загрузки:

IP : 54.196 .121 .252

26 апреля 2023 г., 16:11:54

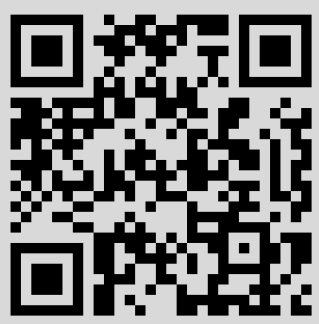




\title{
ФИЗИКА
}

Том 175, № 3

июнь, 2013

(C) 2013 г.

\author{
Ю. Хонконен
}

\section{КОНТУРНО УПОРЯДОЧЕННЫЕ ФУНКЦИИ ГРИНА В СТОХАСТИЧЕСКОЙ ТЕОРИИ ПОЛЯ}

\begin{abstract}
Приведен краткий обзор вопроса о функциональной формулировке теории возмущений для разнообразных функций Грина в квантовой теории поля. В частности, обсуждается контурно упорядоченное представление функций Грина при конечной температуре. Показано, что разложение зависящих от времени функций Грина по теории возмущений при конечной температуре можно построить с помощью стандартного правила Вика в функциональном виде без введения комплексного времени и эволюции вспять по времени. Обсуждается проблема факторизации соответствующего функционального интеграла. Функции Грина решения стохастических дифференциальных уравнений построены в форме Швингера-Келдыша с представлением в виде функционального интеграла с явно сплетающимися физическими и вспомогательными полями.
\end{abstract}

Ключевые слова: функции Грина, температурные функции Грина, временны́е функции Грина при конечной температуре, функциональное представление, функциональный интеграл, стохастическое дифференциальное уравнение.

DOI: $10.4213 / \operatorname{tmf} 8483$

\section{1. ВВЕДЕНИЕ}

Функции Грина относятся к наиболее важным средствам среди методов квантовой теории поля, применяемых в различных областях физики и в других системах, описываемых в терминах подходящих математических моделей. Общее определение функции Грина в квантовой теории - это среднее значение упорядоченного по времени произведения операторов Гейзенберга:

$$
G_{n}\left(x_{1}, x_{2}, \ldots, x_{n}\right)=\operatorname{Tr}\left\{\widehat{\rho} T\left[\widehat{\varphi}_{\mathrm{H}}\left(x_{1}\right) \widehat{\varphi}_{\mathrm{H}}\left(x_{2}\right) \ldots \widehat{\varphi}_{\mathrm{H}}\left(x_{n}\right)\right]\right\} \text {. }
$$

Зависящие от времени операторы в картине Гейзенберга в большом каноническом ансамбле системы, описываемой оператором Гамильтона $\widehat{H}$, определяются как

$$
\widehat{\varphi}_{\mathrm{H}}(t, \mathbf{x})=e^{i t(\widehat{H}-\mu \widehat{N}) / \hbar} \widehat{\varphi}(\mathbf{x}) e^{-i t(\widehat{H}-\mu \widehat{N}) / \hbar} .
$$

*National Defence University, Helsinki, Finland. E-mail: juha.honkonen@helsinki.fi 
При нулевой температуре оператор плотности $\widehat{\rho}$ в (1) строится с помощью основного состояния гамильтониана $\widehat{H}$ :

$$
\widehat{\rho}=\mid 0)(0|, \quad \widehat{H}| 0)=0 .
$$

При конечной температуре $T>0$ обычно выбирается оператор плотности в виде

$$
\widehat{\rho}=\frac{e^{-\beta(\widehat{H}-\mu \widehat{N})}}{\operatorname{Tr} e^{-\beta(\widehat{H}-\mu \widehat{N})}} .
$$

Для разложения по теории возмущений представление взаимодействия вводится с помощью оператора эволюции, порожденной свободным оператором Гамильтона $\widehat{H}_{0}$ :

$$
\widehat{\varphi}(t, \mathbf{x})=e^{i t\left(\widehat{H}_{0}-\mu \widehat{N}\right) / \hbar} \widehat{\varphi}(\mathbf{x}) e^{-i t\left(\widehat{H}_{0}-\mu \widehat{N}\right) / \hbar} .
$$

Функции Грина (1) в терминах операторов Дирака (4) имеют вид

$$
G_{n}\left(x_{1}, x_{2}, \ldots, x_{n}\right)=\operatorname{Tr}\left\{\widehat{\rho} \widehat{U}\left(0, t_{\mathrm{f}}\right) T\left[\widehat{\varphi}\left(x_{1}\right) \widehat{\varphi}\left(x_{2}\right) \ldots \widehat{\varphi}\left(x_{n}\right) \widehat{U}\left(t_{\mathrm{f}}, t_{\mathrm{i}}\right)\right] \widehat{U}\left(t_{\mathrm{i}}, 0\right)\right\}
$$

где $t_{\mathrm{f}}>t_{n}>\cdots>t_{2}>t_{1}>t_{\mathrm{i}}$, а оператор эволюции $\widehat{U}$ равен

$$
\widehat{U}\left(t, t^{\prime}\right)=e^{i t\left(\widehat{H}_{0}-\mu \widehat{N}\right) / \hbar} e^{-i\left(t-t^{\prime}\right)(\widehat{H}-\mu \widehat{N}) / \hbar} e^{-i t^{\prime}\left(\widehat{H}_{0}-\mu \widehat{N}\right) / \hbar} .
$$

Именно с операторами эволюции из выражения (5), вынесенными из $T$-произведения, связаны сложности в разложении по теории возмущений.

При $T=0$ все упрощается в силу теоремы Гелл-Мана-Лоу. Адиабатические включение и выключение взаимодействия в прошлом и будущем заменяют основное состояние взаимодействующей системы аналогичным состоянием свободной системы, поскольку

$$
\left.\left.\widehat{U}\left(t_{\mathrm{f}}, 0\right) \mid 0\right) \underset{t_{\mathrm{f}} \rightarrow \infty}{\sim} \beta\left(t_{\mathrm{f}}\right)|0\rangle, \quad \widehat{U}\left(t_{\mathrm{i}}, 0\right) \mid 0\right) \underset{t_{\mathrm{i}} \rightarrow-\infty}{\sim} \alpha\left(t_{\mathrm{i}}\right)|0\rangle .
$$

Фазовые множители $\alpha\left(t_{\mathrm{i}}\right)$ и $\beta\left(t_{\mathrm{f}}\right)$ определяют нормировку, а функции Грина $(1)$ становятся равными средним значениям по основному состоянию свободной системы:

$$
\left(0\left|T\left[\widehat{\varphi}_{\mathrm{H}}\left(x_{1}\right) \widehat{\varphi}_{\mathrm{H}}\left(x_{2}\right) \ldots \widehat{\varphi}_{\mathrm{H}}\left(x_{n}\right)\right]\right| 0\right)=\lim _{\substack{t_{\mathrm{f}} \rightarrow \infty \\ t_{\mathrm{i}} \rightarrow-\infty}} \frac{\left\langle 0\left|T\left[\widehat{\varphi}\left(x_{1}\right) \widehat{\varphi}\left(x_{2}\right) \ldots \widehat{\varphi}\left(x_{n}\right) \widehat{U}\left(t_{\mathrm{f}}, t_{\mathrm{i}}\right)\right]\right| 0\right\rangle}{\left\langle 0\left|\widehat{U}\left(t_{\mathrm{f}}, t_{\mathrm{i}}\right)\right| 0\right\rangle}
$$

Для такого матричного элемента разложение по теории возмущений можно построить с помощью теорем Вика, которые особенно удобны в функциональном виде, как это подробно изложено в классической монографии [1].

При конечной температуре $T>0$ для зависящих от времени операторов вида

$$
\begin{aligned}
\widehat{\varphi}_{\mathrm{M}}(t, \mathbf{x}) & =e^{t(\widehat{H}-\mu \widehat{N}) / \hbar} \widehat{\varphi}(\mathbf{x}) e^{-t(\widehat{H}-\mu \widehat{N}) / \hbar} \\
\widehat{\varphi}_{\mathrm{e}}(t, \mathbf{x}) & =e^{t\left(\widehat{H}_{0}-\mu \widehat{N}\right) / \hbar} \widehat{\varphi}(\mathbf{x}) e^{-t\left(\widehat{H}_{0}-\mu \widehat{N}\right) / \hbar}
\end{aligned}
$$

где $\widehat{\varphi}_{\mathrm{M}}, \widehat{\varphi}_{\mathrm{e}}-$ соответственно евклидовы операторы Гейзенберга и Дирака, часто используется трюк Мацубары с эволюцией по "мнимому времени". 
Если временнь́е аргументы полевых операторов в функции Грина удовлетворяют условию $0<t<\beta \hbar$, то в евклидовом эволюционном операторе начальный момент времени в (5) можно выбрать как $t_{\mathrm{i}}=0$, а конечный - как $t_{\mathrm{f}}=\hbar \beta$. В этом случае евклидов оператор эволюции

$$
\widehat{U}_{\mathrm{e}}\left(t, t^{\prime}\right)=e^{t\left(\widehat{H}_{0}-\mu \widehat{N}\right) / \hbar} e^{-\left(t-t^{\prime}\right)(\widehat{H}-\mu \widehat{N}) / \hbar} e^{-t^{\prime}\left(\widehat{H}_{0}-\mu \widehat{N}\right) / \hbar}
$$

дает эволюционную экспоненту $\widehat{U}_{\mathrm{e}}(0, \beta \hbar)=e^{\beta(\widehat{H}-\mu \widehat{N}) / \hbar} e^{-\beta\left(\widehat{H}_{0}-\mu \widehat{N}\right) / \hbar}$, которая преобразует оператор плотности большого канонического ансамбля взаимодействующей системы в аналогичный оператор для свободной системы. Поэтому для функций Грина в представлении Мацубары мы получаем следующие средние значения в свободной системе:

$$
\begin{aligned}
G_{n}\left(x_{1}, x_{2}, \ldots, x_{n}\right) & =\frac{1}{Z} \operatorname{Tr}\left\{e^{-\beta(\widehat{H}-\mu \widehat{N}) / \hbar} T\left[\widehat{\varphi}_{\mathrm{M}}\left(x_{1}\right) \widehat{\varphi}_{\mathrm{M}}\left(x_{2}\right) \ldots \widehat{\varphi}_{\mathrm{M}}\left(x_{n}\right)\right]\right\}= \\
& =\frac{1}{Z} \operatorname{Tr}\left\{e^{-\beta\left(\widehat{H}_{0}-\mu \widehat{N}\right) / \hbar} T\left[\widehat{\varphi}_{\mathrm{e}}\left(x_{1}\right) \widehat{\varphi}_{\mathrm{e}}\left(x_{2}\right) \ldots \widehat{\varphi}_{\mathrm{e}}\left(x_{n}\right) \widehat{U}(\beta \hbar, 0)\right]\right\}
\end{aligned}
$$

Правила Фейнмана в основном те же самые, что и в случае функций Грина при нулевой температуре. Важные различия, однако, заключаются в евклидовой эволюции полевых операторов и добавлении теплового среднего нормального произведения двух полевых операторов к пропагаторам модели (см., например, [1]).

Наиболее сложным является случай зависящих от времени функций Грина при конечной температуре, где временна́я эволюция порождается обычным уравнением Шредингера, а в определении (1) выбирается оператор плотности большого канонического ансамбля. Представление теории возмущений для таких функций Грина в литературе обычно основано на идее Швингера [2] о контурно упорядоченном произведении операторов в комплексной временной плоскости [3], [4] вместо обычного упорядоченного по времени произведения в (1).

Кроме обычных квантово-механических задач, важные стохастические задачи можно сформулировать в терминах полевых операторов, аналогичных операторам квантовой теории поля [5], [6], которые порождают функции Грина, определенные по аналогии с (1). Основное отличие от функций Грина квантовой теории поля состоит в виде оператора плотности, который в случае стохастических задач построен существенно отличающимся от (2) или (3) путем.

В настоящей работе автор ставил две цели. Во-первых, показать, что остроумная идея использования контурно упорядоченного произведения для вычисления зависящих от времени функций Грина в теории возмущений на самом деле больше не нужна. Разложение по теории возмущений этих функций Грина несложно получить с помощью стандартных теорем Вика, если их применять в компактном и ясном функциональном виде, который использовался в работе [1]. В частности, нам не нужны ни комплексные временнь́е переменные, ни какая бы то ни было обратная временна́я эволюция полевых операторов. Во-вторых, обсудить применение зависящих от времени функций Грина к анализу стохастических задач на примере стохастического дифференциального уравнения (СДУ) второго порядка. 


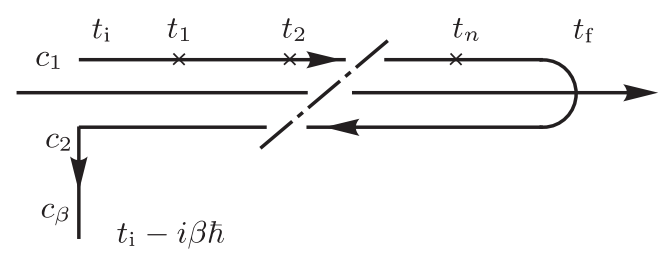

Рис. 1. Контур для контурно упорядоченной функции Грина. Часть контура, начинающаяся при $t_{\mathrm{i}}$ и продолжающаяся до $t_{\mathrm{f}}$, обозначена как $c_{1}$ (эта часть соответствует физическим моментам времени). Через $c_{2}$ обозначена часть контура от $t_{\mathrm{f}}$ до $t_{\mathrm{i}}$, а $c_{\beta}$ - часть контура от $t_{\mathrm{i}}$ вдоль мнимой оси. Полный контур обозначен как $c=c_{1} \cup c_{2} \cup c_{\beta}$.

Работа построена следующим образом. В разделе 2 приведен краткий обзор контурно упорядоченных функций Грина. Постороение разложения по теории возмущений для зависящих от времени функций Грина при конечной температуре с использованием только стандартных теорем Вика проведено в разделе 3. Решение СДУ второго порядка в терминах функций Грина представлено в разделе 4. Раздел 5 содержит заключение и обсуждение результатов.

\section{2. КОНТУРНО УПОРЯДОЧЕННЫЕ ФУНКЦИИ ГРИНА}

Среднее значение контурно упорядоченного произведения $T_{\mathrm{c}}$ операторов Гейзенберга

$$
G_{n}\left(x_{1}, x_{2}, \ldots, x_{n}\right)=\operatorname{Tr}\left\{\widehat{\rho} T_{\mathrm{c}}\left[\widehat{\varphi}_{\mathrm{H}}\left(x_{1}\right) \widehat{\varphi}_{\mathrm{H}}\left(x_{2}\right) \ldots \widehat{\varphi}_{\mathrm{H}}\left(x_{n}\right)\right]\right\}
$$

представляет собой контурно упорядоченную функцию Грина. Контурно упорядоченное произведение является обобщением обычного хронологического произведения, в котором зависящие от времени операторы расставлены в порядке их временнь́х аргументов на снабженном направлением (замкнутом) контуре на комплексной временно́й плоскости. Предполагается, что оператор плотности $\widehat{\rho}$ в (7) описывает начальное распределение вероятности на контуре.

Операторная экспонента в операторе плотности для большого канонического ансамбля обычно включается в контур на комплексной временной плоскости с использованием представления Мацубары, что дает контур, приведенный на рис. 1. Предполагается, что контурно упорядоченная функция Грина, соответствующая этому контуру $c=c_{1} \cup c_{2} \cup c_{\beta}$, в представлении взаимодействия имеет вид $\left(\widehat{V}=\widehat{H}-\widehat{H}_{0}\right)$

$$
\begin{aligned}
G_{n}\left(x_{1}, x_{2}, \ldots, x_{n}\right)= & \frac{1}{Z} \operatorname{Tr}\left\{e ^ { - \beta ( \widehat { H } _ { 0 } - \mu \widehat { N } ) / \hbar } T _ { \mathrm { c } } \left[\widehat{\varphi}\left(x_{1}\right) \widehat{\varphi}\left(x_{2}\right) \ldots \widehat{\varphi}\left(x_{n}\right) \times\right.\right. \\
& \left.\left.\times \exp \left(-i \int_{c} d t \widehat{V}(t)\right)\right]\right\} .
\end{aligned}
$$

Отметим отсутствие отдельных эволюционных операторов вне $T_{\mathrm{c}}$-произведения.

В большинстве случаев пределы $t_{\mathrm{f}} \rightarrow \infty$ и $t_{\mathrm{i}} \rightarrow-\infty$ берутся при допущении, что система "забыла" обо всех начальных корреляциях. В этом случае за начальное распределение вероятности в (7) проще всего взять большое каноническое распределение для свободной системы и получить представление Келдыша для функций 


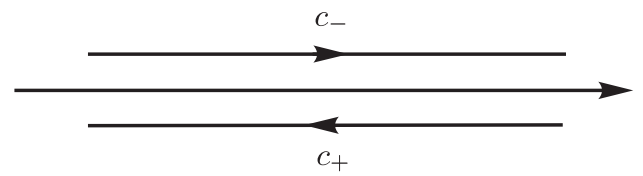

Рис. 2. Контур Келдыша для функций Грина. Физические временны́е переменные берутся на контуре $c_{-}$, а временны́е переменные на контуре $c_{+}$ появляются во внутренней структуре графов теории возмущений.

Грина [7] на контуре Келдыша, приведенном на рис. 2. В подходе Келдыша двухточечные функции Грина ставятся в соответствие с одночастичными функциями плотности вероятности в “фазовом пространстве" с помощью представления функции Вигнера. Тогда уравнение Дайсона для полных пропагаторов можно рассматривать как квантовое кинетическое уравнение [7].

\section{3. ЗАВИСЯЩИЕ ОТ ВРЕМЕНИ ФУНКЦИИ ГРИНА ПРИ КОНЕЧНОЙ ТЕМПЕРАТУРЕ}

Построить зависящие от времени функции Грина можно, однако, и вполне традиционным (хотя и менее эффектным) способом с помощью теорем Вика для симметричных произведений, как это было сделано в работе [1] при построении функций Грина при нулевой температуре и функций Мацубары.

Начнем с обычного выражения для зависящих от времени функций Грина при $T>0$ в представлении Дирака:

$$
\begin{aligned}
G_{n}\left(x_{1}, x_{2}, \ldots, x_{n}\right)= & \frac{1}{\operatorname{Tr} e^{-\beta \widehat{H}}} \operatorname{Tr}\left\{e^{-\beta \widehat{H}_{0}} \widehat{U}\left(-i \beta \hbar+t_{\mathrm{i}}, t_{\mathrm{i}}\right) \widehat{U}\left(t_{\mathrm{i}}, t_{\mathrm{f}}\right) \times\right. \\
& \left.\times T\left[\widehat{\varphi}\left(x_{1}\right) \widehat{\varphi}\left(x_{2}\right) \ldots \widehat{\varphi}\left(x_{n}\right) \exp \left[-\frac{i}{\hbar} \int_{t_{\mathrm{i}}}^{t_{\mathrm{f}}} \widehat{V}(u) d u\right]\right]\right\} .
\end{aligned}
$$

В выражении (9) оператор эволюции $\widehat{U}\left(-i \beta \hbar+t_{\mathrm{i}}, t_{\mathrm{i}}\right)$, аргументом которого является комплексное время, формально является сокращенным представлением произведения обыкновенных (свободных) и евклидовых операторов эволюции:

$$
\widehat{U}\left(-i \beta \hbar+t_{\mathrm{i}}, t_{\mathrm{i}}\right)=e^{i \widehat{H}_{0} t_{\mathrm{i}} / \hbar} \widehat{U}_{\mathrm{e}}(\beta \hbar, 0) e^{-i \widehat{H}_{0} t_{\mathrm{i}} / \hbar} .
$$

Обычная упорядоченная по времени экспонента для оператора эволюции

$$
\widehat{U}\left(t_{\mathrm{f}}, t_{\mathrm{i}}\right)=T \exp \left[-\frac{i}{\hbar} \int_{t_{\mathrm{i}}}^{t_{\mathrm{f}}} \widehat{V}(t) d t\right]
$$

уже была введена в $T$-произведении (9). Введем аналогичное представление для евклидова оператора эволюции, а также для оператора

$$
\widehat{U}_{\mathrm{e}}(\beta \hbar, 0)=T \exp \left[-\frac{1}{\hbar} \int_{0}^{\beta \hbar} \widehat{V}_{\mathrm{e}}(t) d t\right]
$$


где $\widehat{V}_{\mathrm{e}}(t)$ берется в картине евклидова взаимодействия (6). Для оператора эволюции $\widehat{U}\left(t_{\mathrm{i}}, t_{\mathrm{f}}\right)$ из-за наличия упорядоченных по времени аргументов $\left(t_{\mathrm{f}}>t_{\mathrm{i}}\right)$ представление, полученное с использованием антихронологически упорядоченной экспоненты, имеет вид

$$
\widehat{U}\left(t_{\mathrm{i}}, t_{\mathrm{f}}\right)=\widetilde{T} \exp \left[\frac{i}{\hbar} \int_{t_{\mathrm{i}}}^{t_{\mathrm{f}}} \widehat{V}(u) d u\right]
$$

где антихронологическое произведение $\widetilde{T}$ располагает операторы в порядке возрастания времени слева направо. $\widetilde{T}$-произведение порождает антихронологическую свертку, определяемую как $\widetilde{T}\left[\widehat{\varphi}(t) \widehat{\varphi}\left(t^{\prime}\right)\right]=N\left[\widehat{\varphi}(t) \widehat{\varphi}\left(t^{\prime}\right)\right]+\tilde{\Delta}\left(t, t^{\prime}\right)$. Таким образом, функция Грина выражается (с использованием сокращенного обозначения (10)) как

$$
\begin{aligned}
G_{n}\left(x_{1}, x_{2}, \ldots, x_{n}\right) & =\frac{1}{\operatorname{Tr} e^{-\beta \widehat{H}}} \operatorname{Tr}\left(e^{-\beta \widehat{H}_{0}} T_{c_{\beta}} \exp \left[-\frac{i}{\hbar} \int_{c_{\beta}} \widehat{V}(u) d u\right] \times\right. \\
& \left.\times \widetilde{T} \exp \left[\frac{i}{\hbar} \int_{t_{\mathrm{i}}}^{t_{\mathrm{f}}} \widehat{V}(u) d u\right] T\left\{\widehat{\varphi}\left(t_{1}\right) \widehat{\varphi}\left(t_{2}\right) \ldots \widehat{\varphi}\left(t_{n}\right) \exp \left[-\frac{i}{\hbar} \int_{t_{\mathrm{i}}}^{t_{\mathrm{f}}} \widehat{V}(u) d u\right]\right\}\right) .
\end{aligned}
$$

Здесь теоремы Вика позволяют слить произведение упорядоченных по времени произведений в одно нормально упорядоченное произведение [1], в результате чего получаем выражение

$$
\begin{aligned}
G_{n}\left(x_{1}, \ldots, x_{n}\right)= \\
=\frac{1}{Z} \operatorname{Tr}\left(e ^ { - \beta \widehat { H } _ { 0 } } N \left\{\exp \left(\frac{1}{2} \sum_{l=1}^{3} \frac{\delta}{\delta \varphi_{l}} \Delta_{l l} \frac{\delta}{\delta \varphi_{l}}+\sum_{k>l} \frac{\delta}{\delta \varphi_{k}} n_{k l} \frac{\delta}{\delta \varphi_{l}}\right) \varphi_{1}\left(x_{1}\right) \ldots \varphi_{1}\left(x_{n}\right) \times\right.\right. \\
\left.\left.\quad \times \exp \left[-\frac{i}{\hbar} \int_{c_{\beta}} V\left(\varphi_{3}\right) d t+\frac{i}{\hbar} \int_{t_{\mathrm{i}}}^{t_{\mathrm{f}}} V\left(\varphi_{2}\right) d t-\frac{i}{\hbar} \int_{t_{\mathrm{i}}}^{t_{\mathrm{f}}} V\left(\varphi_{1}\right) d t\right]\right\}\left.\right|_{\varphi_{i}=\widehat{\varphi}}\right) .
\end{aligned}
$$

Среднее значение нормального произведения любого операторного функционала $F(\widehat{\varphi})$ вычисляется с помощью соотношения

$$
\frac{1}{\operatorname{Tr} e^{-\beta \widehat{H}_{0}}} \operatorname{Tr}\left\{e^{-\beta \widehat{H}_{0}} N[F(\widehat{\varphi})]\right\}=\left.\exp \left(\frac{1}{2} \frac{\delta}{\delta \varphi} d \frac{\delta}{\delta \varphi}\right) F(\varphi)\right|_{\varphi=0}
$$

где ядро (которое нужно прибавить к пропагаторной матрице в (11)) равно

$$
d\left(x, x^{\prime}\right)=\frac{1}{\operatorname{Tr} e^{-\beta \widehat{H}_{0}}} \operatorname{Tr}\left\{e^{-\beta \widehat{H}_{0}} N\left[\widehat{\varphi}(x) \widehat{\varphi}\left(x^{\prime}\right)\right]\right\}
$$

Однако следует иметь в виду, что в (11) имеются поля, подчиняющиеся и евклидовой, и псевдоевклидовой эволюции, которые возникают из-за различных полевых операторов. Поэтому тепловые средние значения (12) образуют матрицу, элементы которой прибавляются к соответствующим пропагаторам и нормальным сверткам в (11). 
Таким образом, функциональное представление, дающее разложение по теории возмущений для зависящих от времени функций Грина при $T>0$, имеет вид

$$
\begin{aligned}
& G_{n}\left(x_{1}, \ldots, x_{n}\right)=\frac{Z_{0}}{Z} \exp \left(\frac{1}{2} \sum_{l=1}^{3} \frac{\delta}{\delta \varphi_{l}} \Delta_{l l} \frac{\delta}{\delta \varphi_{l}}+\right. \\
& \left.\quad+\sum_{k>l} \frac{\delta}{\delta \varphi_{k}} n_{k l} \frac{\delta}{\delta \varphi_{l}}+\frac{1}{2} \sum_{k, l} \frac{\delta}{\delta \varphi_{k}} d_{k l} \frac{\delta}{\delta \varphi_{l}}\right) \varphi_{1}\left(x_{1}\right) \ldots \varphi_{1}\left(x_{n}\right) \times \\
& \quad \times\left.\exp \left[-\frac{i}{\hbar} \int_{c_{\beta}} V\left(\varphi_{3}\right) d t+\frac{i}{\hbar} \int_{t_{\mathrm{i}}}^{t_{\mathrm{f}}} V\left(\varphi_{2}\right) d t-\frac{i}{\hbar} \int_{t_{\mathrm{i}}}^{t_{\mathrm{f}}} V\left(\varphi_{1}\right) d t\right]\right|_{\varphi_{i}=0} .
\end{aligned}
$$

Легко понять, что этот функционал порождает точно такую же теорию возмущений, что и контурно упорядоченная функция Грина (8). Основной момент здесь заключается в том, что для получения представления (13) не надо вводить переменные комплексного времени или обратную эволюции по времени. Функционал (13) получается исключительно с помощью стандартных теорем Вика.

Обычно при переходе от функционального представления типа (13) к представлению в виде функционального интеграла имеется техническая проблема, которая существенно уменьшает пользу от применения функционального интеграла. Чтобы в этом разобраться, рассмотрим в качестве примера слабо взаимодействующий бозонный газ. Пусть взаимодействие локально, а рассматриваемое функциональное представление имеет вид (13) для двухточечной функции Грина

$$
\begin{aligned}
G_{12}\left(x_{1}, x_{2}\right)= & \frac{Z_{0}}{Z} \exp \left(\frac{\delta}{\delta \psi_{1}} \Delta_{\beta} \frac{\delta}{\delta \psi_{1}^{+}}+\frac{\delta}{\delta \psi_{2}} \tilde{\Delta}_{\beta} \frac{\delta}{\delta \psi_{2}^{+}}+\right. \\
& \left.+\frac{\delta}{\delta \psi_{2}} n_{\beta} \frac{\delta}{\delta \psi_{1}^{+}}+\frac{\delta}{\delta \psi_{1}} d \frac{\delta}{\delta \psi_{2}^{+}}\right) \psi_{1}\left(x_{1}\right) \psi_{1}^{+}\left(x_{2}\right) \times \\
& \times \exp \left[\frac{i \lambda}{4 \hbar} \int_{-\infty}^{\infty} d t \int d \mathbf{x}\left(\psi_{2}^{+}\right)^{2} \psi_{2}^{2}-\frac{i \lambda}{4 \hbar} \int_{-\infty}^{\infty} d t \int d \mathbf{x}\left(\psi_{1}^{+}\right)^{2} \psi_{1}^{2}\right]_{\psi_{i}^{ \pm}=0}
\end{aligned}
$$

с пропагаторами $\Delta_{\beta}=\Delta+d, \tilde{\Delta}_{\beta}=\tilde{\Delta}+d$ и $n_{\beta}=n+d$. Функциональный интеграл введен в (14) путем выражения экспоненты функционального дифференциального оператора второго порядка в правой части в виде гауссова функционального интеграла с экспонентой от линейных дифференциальных операторов [1]. Функционал взаимодействия сохраняет вид, приведенный в (14), при этом для действия свободных полей получаем

$$
S_{0}=\int d t \int d \mathbf{x}\left[\psi_{1}^{+}\left(i \hbar \frac{\partial}{\partial t}+\frac{\hbar^{2} \nabla^{2}}{2 m}+\mu\right) \psi_{1}-\psi_{2}^{+}\left(i \hbar \frac{\partial}{\partial t}+\frac{\hbar^{2} \nabla^{2}}{2 m}+\mu\right) \psi_{2}\right] .
$$

Здесь и во взаимодействии (14) нет явной связи между физическими полями $\psi_{1}^{ \pm}$и вспомогательными полями $\psi_{2}^{ \pm}$, и интеграл для вспомогательных полей (формально) факторизуется! То, что пренебрежение вспомогательным полем приводит к неопределенностям в теории возмущений, было замечено уже довольно давно [8], и проблема функционального интеграла заключается в том, что он не отражает однозначно структуру пропагаторов, которая явно выражена в функционале (14). 


\section{4. ТЕОРИЯ ПОЛЯ ДЛЯ СТОХАСТИЧЕСКОГО ДИФФЕРЕНЦИАЛЬНОГО УРАВНЕНИЯ ВТОРОГО ПОРЯДКА}

Наличие случайности, внутренне присущей различным моделям, описывающим эволюцию физических, химических, биологических, социальных и финансовых процессов, учитывается наиболее непосредственно при помощи дифференциальных уравнений со случайными коэффициентными функциями (источниками), т. е. СДУ. Такой метод позволяет описывать флуктуации с помощью уравнения Ланжевена

$$
\frac{\partial \varphi}{\partial t}=-K \varphi+U(\varphi)+f b(\varphi), \quad\left\langle f(t) f\left(t^{\prime}\right)\right\rangle=\delta\left(t-t^{\prime}\right) D .
$$

СДУ с белым шумом (15) является математически некорректно определенным. Способ непосредственного получения математически непротиворечивого уравнения состоит в использовании $\delta$-последовательности с последующим переходом к $\delta$-функциональному пределу:

$$
\left\langle f(t, \mathbf{x}) f\left(t^{\prime}, \mathbf{x}^{\prime}\right)\right\rangle=\bar{D}\left(t, \mathbf{x} ; t^{\prime}, \mathbf{x}^{\prime}\right) \rightarrow \delta\left(t-t^{\prime}\right) D\left(\mathbf{x}, \mathbf{x}^{\prime}\right), \quad t^{\prime} \rightarrow t,
$$

что приводит к интерпретации СДУ в форме Стратоновича (15) (см., например, [9]). Однако как в теоретическом анализе, так и в теории возмущений более удобной является форма Ито, хотя ее не так легко получить из-за проблемы с конечным временем корреляции.

СДУ первого порядка представляют собой стандартный инструмент при анализе флуктуаций. Здесь мы обсудим СДУ второго порядка

$$
m \frac{\partial^{2} \varphi}{\partial t^{2}}+\beta \frac{\partial \varphi}{\partial t}=-K \varphi+U(\varphi)+f b(\varphi) .
$$

Удобно представить задачу (16) в виде системы двух уравнений первого порядка для двойного набора переменных:

$$
\frac{\partial \varphi}{\partial t}=v, \quad m \frac{\partial v}{\partial t}+\beta v=-K \varphi+U(\varphi)+f b(\varphi) .
$$

Для функции плотности условной вероятности данной задачи $p\left(t, \varphi, v \mid t_{0}, \varphi_{0}, v_{0}\right)$ стандартные правила дают уравнение Фоккера-Планка (УФП)

$$
\frac{\partial}{\partial t} p=-\frac{\partial}{\partial \varphi}(v p)+\frac{\partial}{\partial v}\left[\left(\frac{\beta}{m} v+\frac{K}{m} \varphi-\frac{U}{m}\right) p\right]+\frac{1}{2} \frac{\partial^{2}}{\partial v^{2}}[b D b p]
$$

где принята интерпретация Ито для СДУ (17).

Используя аналогию между УФП и уравнением Шредингера, решение УФП можно представить аналогично тому, как это делается в квантовой теории поля [6]. Если использовать операторы

$$
\widehat{\varphi}, \widehat{\pi}=-\frac{\partial}{\partial \varphi}, \quad[\widehat{\varphi}, \widehat{\pi}]=1 ; \quad \widehat{v}, \widehat{\eta}=-\frac{\partial}{\partial v}, \quad[\widehat{v}, \widehat{\eta}]=1,
$$

то УФП (18) для вектора состояния $\left\langle\varphi, v \mid p_{t}\right\rangle:=p(\varphi, v, t)$ примет вид

$$
\frac{\partial}{\partial t}\left|p_{t}\right\rangle=\hat{L}\left|p_{t}\right\rangle, \quad \hat{L}=\widehat{\pi} \widehat{v}-\widehat{\eta}\left[\frac{\beta}{m} \widehat{v}+\frac{K}{m} \widehat{\varphi}-\frac{U(\widehat{\varphi})}{m}\right]+\frac{1}{2} \widehat{\eta}^{2} b(\widehat{\varphi}) D b(\widehat{\varphi}) .
$$


Зависящие от времени операторы в представлении Гейзенберга и в представлении Дирака вводятся по аналогии с евклидовой теорией поля:

$$
\widehat{\varphi}_{\mathrm{H}}(t)=e^{-\hat{L} t} \widehat{\varphi} e^{\hat{L} t}, \quad \widehat{\varphi}(t)=e^{-\hat{L}_{0} t} \widehat{\varphi} e^{\hat{L}_{0} t}, \quad \hat{L}_{0}=\widehat{\pi} \widehat{v}-\frac{\beta}{m} \widehat{\eta} \widehat{v} .
$$

Производящая функция

$$
G(J)=\operatorname{Tr} \widehat{\rho}_{0} T\left[\exp \left(\int_{t_{\mathrm{i}}}^{t_{\mathrm{f}}} d t \widehat{\varphi}_{\mathrm{H}}(t) J(t)\right)\right], \quad \widehat{\rho}_{0}=\iint d \varphi d v\left|p_{0}\right\rangle\langle\varphi, v|,
$$

вычисляется по аналогии с евклидовой теорией поля. В представлении Дирака $\left(\hat{L}=\hat{L}_{0}+\hat{L}_{\mathrm{I}}, t_{\mathrm{f}}>t_{\mathrm{i}}>t_{0}\right)$ получаем

$$
\begin{aligned}
& T \exp \left(\int \widehat{\varphi}_{\mathrm{H}} J d t\right)=e^{\hat{L}_{0} t_{0}} \widehat{U}\left(t_{0}, t_{\mathrm{f}}\right) T\left[\exp \left(\int \widehat{\varphi} J d t+\widehat{S}_{\mathrm{I}}\right)\right] \widehat{U}\left(t_{\mathrm{i}}, t_{0}\right) e^{-\hat{L}_{0} t_{0}}= \\
& =e^{\hat{L}_{0} t_{0}} \widetilde{T} \exp \left(-\int_{t_{0}}^{t_{\mathrm{f}}} \hat{L}(t) d t\right) T\left[\exp \left(\int \widehat{\varphi} J d t+\widehat{S}_{\mathrm{I}}\right)\right] T \exp \left(\int_{t_{0}}^{t_{\mathrm{i}}} \hat{L}(t) d t\right) e^{-\hat{L}_{0} t_{0}}
\end{aligned}
$$

где $\widehat{U}\left(t, t^{\prime}\right)=e^{-t \hat{L}_{0}} e^{\left(t-t^{\prime}\right) \hat{L}} e^{t^{\prime} \hat{L}_{0}}$ и $\widehat{S}_{\mathrm{I}}=\int_{t_{\mathrm{i}}}^{t_{\mathrm{f}}} \hat{L}_{\mathrm{I}}(t) d t$. Хронологические произведения в (21) сливаются в силу теорем Вика в нормальное произведение. Для любого операторного функционала $F[\widehat{\pi}, \widehat{\varphi}, \widehat{\eta}, \widehat{v}]$ вычисление дает [1]

$$
\operatorname{Tr} e^{-\hat{L}_{0} t_{0}} \widehat{\rho}_{0} e^{\hat{L}_{0} t_{0}} N\{F[\widehat{\pi}, \widehat{\varphi}, \widehat{\eta}, \widehat{v}]\}=\iint \mathcal{D} \varphi \mathcal{D} v p_{0}(\varphi, v) F\left[0, n_{1} \varphi+n_{2} v, 0, n_{3} v\right],
$$

где $n_{1}=[\widehat{\varphi}, \widehat{\pi}], n_{2}=[\widehat{\varphi}, \widehat{\eta}]$ и $n_{3}=[\widehat{v}, \widehat{\eta}]$. Отсюда получаем функциональный вид производящей функции (20):

$$
\begin{aligned}
G(J)= & \iint \mathcal{D} \varphi \mathcal{D} v p_{0}(\varphi, v) \exp \left[\frac{\delta}{\delta \varphi_{2}} \tilde{\Delta} \frac{\delta}{\delta \eta_{2}}+\frac{\delta}{\delta \varphi_{1}} \Delta \frac{\delta}{\delta \eta_{1}}+\frac{\delta}{\delta \varphi_{2}} n_{2} \frac{\delta}{\delta \eta_{1}}\right] \times \\
& \times\left.\exp \left[\int_{t_{\mathrm{i}}}^{t_{\mathrm{f}}} \varphi_{1} J d t-\int_{t_{0}}^{t_{\mathrm{f}}} L_{\mathrm{I}}\left(\eta_{2}, \varphi_{2}\right) d t+\int_{t_{0}}^{t_{\mathrm{f}}} L_{\mathrm{I}}\left(\eta_{1}, \varphi_{1}\right) d t\right]\right|_{\substack{\eta_{i}=0 \\
\varphi_{i}=n_{1} \varphi+n_{2} v}} .
\end{aligned}
$$

Стандартное введение функционального интеграла путем представления его как гауссова интеграла для дифференциального оператора в правой части (22) также приводит к факторизации вспомогательных переменных.

Однако в (22) функционал взаимодействия $L_{\mathrm{I}}$ является квадратичным по $\eta$. Поэтому экспоненту от $L_{\mathrm{I}}$ можно записать как гауссов интеграл с экспонентой, линейной по $\eta$, что позволяет вычислить функциональные производные в замкнутом виде и получить (для простоты полагаем, что функция $b$ не зависит от полей)

$$
\begin{aligned}
G(J)= & \iiint \mathcal{D} \varphi \mathcal{D} \xi_{1} \mathcal{D} \xi_{2} p_{0}(\varphi, v) \exp \left\{J\left(\Delta \xi_{1}+n_{1} \varphi+n_{2} v\right)+\right. \\
+ & \frac{1}{2} \xi_{1}(b D b)^{-1} \xi_{1}-\frac{1}{2} \xi_{2}(b D b)^{-1} \xi_{2}+\xi_{2}(b D b)^{-1} U_{2}+\xi_{1}(b D b)^{-1} U_{1}+ \\
& \left.+\frac{1}{2} U_{2}(b D b)^{-1} U_{2}-\frac{1}{2} U_{1}(b D b)^{-1} U_{1}\right\}
\end{aligned}
$$


где $U_{1}=U\left(\Delta \xi_{1}+n_{1} \varphi+n_{2} v\right)$ и $U_{2}=U\left(\tilde{\Delta} \xi_{2}+n_{2} \xi_{1}+n_{1} \varphi+n_{2} v\right)$. В представлении (23) перемешивание переменных теперь присутствует явно, хотя и выглядит довольно непривлекательно. Такое представление приводит к сокращению графов с замкнутыми петлями пропагаторов без явного использования свойства запаздывания пропагаторов.

\section{5. ЗАКЛЮЧЕНИЕ}

В настоящей работе приведено описание вывода функционального представления для функций Грина как для обычных случаев квантовой теории поля, так и для решения СДУ второго порядка. Показано, что контурно упорядоченные функции Грина можно построить как стандартные зависящие от времени функции Грина при конечной температуре с использованием теорем Вика для симметричных произведений. В частности, отмечено, что для получения обычного разложения по теории возмущений не требуется введения комплексного временно́го контура и соответствующего контурно упорядоченного произведения. Также отмечена неопределенность представления в виде функционального интеграла для зависящих от времени функций Грина при конечной температуре.

Построено функциональное представление производящей функции для моментов решения СДУ второго порядка. Чтобы избежать неопределенности, в качестве отправной точки использовалось УФП, соответствующее интерпретации Ито. Показано, что в этом случае структура функционала взаимодействия позволяет построить непротиворечивый функциональный интеграл для производящей функции.

\section{Список литературы}

[1] А.Н. Васильев, Функииональные методы в квантовой теории поля и статистике, ЛГУ, Л., 1976.

[2] J. Schwinger, J. Math. Phys., 2 (1961), 407-432.

[3] J. Rammer, H. Smith, Rev. Modern Phys., 58:2 (1986), 323-359.

[4] N. P. Landsman, Ch. G. van Weert, Phys. Rep., 145:3-4 (1987), 141-249.

[5] M. Doi, J. Phys. A, 9:9 (1976), 1465-1477; 1479-1495.

[6] H. Leschke, M. Schmutz, Z. Phys. B, 27:1 (1977), 85-94.

[7] Л.В. Келдыш, ЖЭЭФ, 47 (1965), 1515-1527.

[8] L. Dolan, R. Jackiw, Phys. Rev. D, 9:12 (1974), 3320-3341.

[9] C. W. Gardiner, Handbook of Stochastic Methods. For Physics, Chemistry and the Natural Sciences, Springer Series in Synergetics, 13, Springer, Berlin, 1985. 